\title{
Konzepte der Filmbildung und der pädagogische Widerwille gegen den Seh-Sinn
}

\section{Ralf Vollbrecht}

\section{Zusammenfassung}

Gängige Konzepte der Filmbildung werden vorgestellt und in ihren pädagogischen Implikationen diskutiert. Dabei wird kritisch auf das Kanon-Konzept eingegangen sowie auf den vom Autor favorisierten Ansatz, Spielfilme auch als Gegenstand einer narrativen Pädagogik zu verstehen.

Kontakt

Prof. Dr. Ralf Vollbrecht ralf.vollbrecht@tu-dresden.de Professor für Medienpädagogik an der Technischen Universität Dresden 


\section{Inhalt}

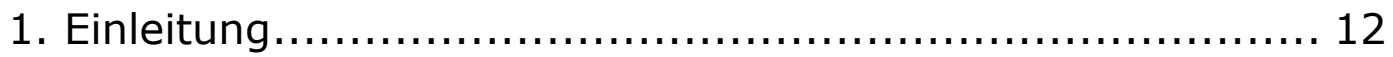

1. Der Filmkanon ............................................... 17

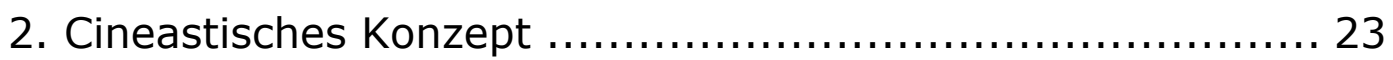

3. Studentisches Kino .......................................... 23

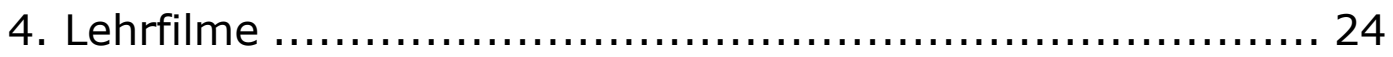

5. Handlungsorientierte Medienpädagogik ........................ 24

6. Der Spielfilm als Lernfeld narrativer Pädagogik ................ 25

7. Filmbildung und Filmverstehen ............................. 28

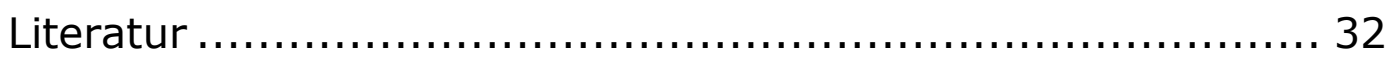




\section{Einleitung}

In den letzten Jahren ist eine erstaunliche Karriere des Begriffs Filmbildung zu beobachten. Das ist insofern verdächtig, zum einen weil die Medienpädagogik in der über 100-jährigen Geschichte des Kinos den Begriff Filmbildung bislang nicht vermisst hat - gleichwohl es von Anfang an das heute mit Filmbildung bezeichnete pädagogische Anliegen und Bemühen gab - und zum anderen, weil auch analoge Begriffsbildungen fehlen. Warum nicht auch Fernsehbildung, Radiobildung, Computerspielbildung oder Comic-Bildung? Hier ginge es doch genauso um das, was für Filmbildung reklamiert wird: ästhetische Erziehung, Medienbildung, die Vermittlung von Kenntnissen für einen kritischen, kreativen und genussvollen Umgang mit diesen Medien.

Die Medienpädagogik tut sich schon schwer mit dem allgemeineren Begriff der Medienbildung, der sich mit dem Bezug auf Bildung ja in eine spezifisch deutsche Denktradition einreiht und nicht nur die Bedeutungsschwere und Problematik dieses Begriffs mitführt, sondern auch an internationale Theoriebildung wenig anschlussfähig ist. Braucht es da weitere Bindestrichbegriffe bzw. Determinativkomposita wie der Filmbildung?

Rekonstruiert man den Diskurs um Filmbildung, so zeigt sich, dass der Begriff nicht die Frucht theoretischer Bemühungen in Medienpädagogik oder Kommunikationswissenschaft ist, sondern als politisches Passepartout geprägt wurde, um im Schulunterricht die Auseinandersetzung mit Filmen zu verankern. Treibende Kraft waren dabei neben der Filmwirtschaft, einigen Kulturinstitutionen und Bildungseinrichtungen vor allem die Bundeszentrale für politische Bildung und die Filmförderungsanstalt (FFA), die 2003 auf dem Kongress Kino macht Schule eine so genannte Filmkompetenzerklärung veröffentlichten, in der die Verankerung von Filmpädagogik in den Lehrplänen gefordert wurde.

Grundsätzlich ist es sehr zu begrüßen, dass Filmpädagogik nun in größerem Umfang in Schulen stattfinden kann. Zugleich ist diese erfreuliche Entwicklung jedoch auch eine nicht unproblematische thematische Verengung des medienpädagogischen Auftrags, denn - anders als aus filmpädagogischer - müsste aus medienpädagogischer Perspektive die Auseinandersetzung mit allen Medien in 
den Schulen erfolgen. Diese Einseitigkeit begünstigt implizit diejenigen filmpädagogischen Ansätze, die den Film als Lehrgegenstand verstehen und für die Schule kanonisieren. Das kann man machen, verfehlt dabei aber möglicherweise die Perspektive der Schülerinnen und Schüler, die mit Filmen heute nicht nur im Kino, sondern in vielen Medien und nicht zuletzt im alltäglichen Gebrauch des Handys rezipierend und aktiv-gestaltend konfrontiert sind.

Der vermeintlich schlichte Begriff Filmbildung hat es also durchaus in sich. Er kann auch ganz unterschiedlich gemeint sein, je nachdem, ob er auf Film betont wird oder auf Bildung, die bei Filmbildung in der linguistischen Konstruktion eines Determinativkompositums ja eingeschränkt wird auf Filmbezüge von Bildung - oder geht es doch um Bildungsbezüge des Films? Und was folgt aus einer solchen Unterscheidung?

Zunächst einmal ist es nicht selbstverständlich, Film oder Medien allgemein mit Bildung zusammen zu denken. In medienpädagogischen oder kommunikationswissenschaftlichen Kreisen mag dies heute selbstverständlich erscheinen - für die Pädagogik insgesamt gilt das nicht und wäre jenseits didaktischer Nützlichkeit in pädagogischen Kontexten noch vor 20 Jahren heftig problematisiert worden - man denke zum Beispiel an die Position eines renommierten Schulpädagogen wie Hartmut von Hentig. Auch heute zeigt die bloße Existenz einer Initiative "Keine Bildung ohne Medien!", dass die Forderung noch nicht eingelöst ist - sonst müsste man sie nicht stellen.

Es gibt offenbar pädagogische Vorbehalte, einen „pädagogische(n) Widerwille(n) gegen den Seh-Sinn", wie Dieter Baacke das einmal genannt hat (Baacke 1995 - die dort vorgetragenen Überlegungen sind teilweise der Veröffentlichung "Treffpunkt Kino. Daten und Materialien zum Verhältnis von Jugend und Kino" (Autoren: Dieter Baacke, Horst Schäfer, Ralf Vollbrecht), Juventa-Verlag Weinheim/München 1994 entnommen). Er vertrat dabei die These, dass das pädagogische Denken und die pädagogische Praxis insbesondere in den Schulen "jede Art von Sinnenerfahrung einzuschränken sucht" - speziell auch das Sehen - zu Gunsten "rationalisierender Abstraktion" (Baacke 1995, S. 83). Diesen Gedanken könnte man anhand der pädagogischen Einschätzungen zum Kino 
in den letzten 100 Jahren weiter entfalten. Das will ich an dieser Stelle nicht tun, sondern nur einige wenige Punkte hervorheben.

In den Anfangsjahren richtete sich die Kritik gegen das Kino sowohl gegen Inhalte und Machart der Filme (Schundfilme) als auch gegen den Ort selbst, gegen das Kino als Ort optischer Verdummung, gegen das Kino als ein Jahrmarkts- und Varietee-Vergnügen für schlichte Gemüter. Diese Orte waren in pädagogischer Hinsicht ebenso wenig Vertrauen erweckend wie das unkontrollierte Beisammensein von Männern, Frauen, Jugendlichen und Kindern beiderlei Geschlechts, die in dunklen Kinoräumen eng beieinander saßen. Heute ist das Kino als Ort jugendlichen Filmvergnügens gesellschaftlich akzeptiert, nicht zuletzt deshalb, weil es unter der kontrollierenden Obhut des Kinder- und Jugendschutzes steht. Aber man muss als Ort nur an die neuen digitalen Räume der Kinder und Jugendlichen denken, die Filme auch im Internet und auf Handys anschauen, und schon ist das Argument pädagogisch wieder ganz modern.

Verdächtig war auch die Schaulust und Faszination, die vom Film ausging. Im Beitrag von Schultze (1911) über den "Kinematograph als Bildungsmittel" klingt das so:

"Da in den Nachmittagsstunden, in denen viele Erwachsene beruflich beschäftigt sind,

während die Kinderwelt ihre Schularbeiten größtenteils erledigt hat, allenthalben große Kindermassen vorhanden sind, die nach einem Vergnügen Ausschau halten, so haben die Kinematographenbesitzer durch billige Kinderkarten ganze Scharen von Kindern anzulocken gewußt. Wie die Motten ins Licht, so flattern sie den Lichtspieltheatern zu. Je greller die Beleuchtung des Eingangs, je grausiger die dort ausgehängten Plakate, desto größer die kindliche Besucherschar. Wer die Seele des Kindes kennt, wird sich darüber nicht wundern - wohl aber darüber, daß man es längere Zeit hindurch ruhig mit angesehen hat, welche Wirkung hier vielfach auf die Seelen von Tausenden und Abertausenden von Kindern ausgeübt wurde." (Schultze 1911, S. 114)

Diese kulturkritische Sicht, die - nebenbei bemerkt im Grundtenor bis in die siebziger Jahre vorherrschend war -, führte damals zu 
einer Fülle von Gegenmaßnahmen, die man heute als pädagogische Interventionsstrategien bezeichnen würde. In der Lehrerschaft gab es jedoch von Anfang an auch Befürworter des Films, die den Film als didaktisches Mittel schätzten, jedenfalls wenn er dokumentarisch war (, statt die Welt zu verfälschen und die Sinne übermäßig zu reizen).

Die Situation änderte sich, als das Bürgertum spätestens in den zwanziger und dreißiger Jahren mit der Ausdifferenzierung der Filmsprache, der Film-Themen, der Film-Ästhetik, den Film auch für sich entdeckte, und repräsentative Film-Paläste entstanden, in die zu gehen man sich nicht schämen musste. In Filmkunst- „Theatern" trifft man nun auch auf Intellektuelle, denn Filme gelten nicht mehr per sie als verdummend, sondern können ebenso eine Kunst sein - Film wird zu einem anerkannten Kulturgut.

Damit hat der Film eine Stufe der gesellschaftlichen Anerkennung erreicht, die Computerspiele gerade eben erst erklimmen. Kulturrat und Kulturstaatsminister haben bekanntlich 2007 Computerspiele als "Kulturgut" bezeichnet - nicht ohne heftigen Widerspruch. Der Generalsekretär des Deutschen Musikrates, Christian Höppner, befand sogleich, es sei "abstrus", Computerspiele zum Kulturgut zu erheben. Und überhaupt sollten Kinder lieber musizieren. So ähnlich darf man sich durchaus die damaligen Debatten zum Film vorstellen, und während wir über Filmbildung diskutieren, sind wir von Computerspiel-Bildung noch weit entfernt. Die Frage ist allerdings, welcher Bildungsbegriff jeweils unterlegt ist.

Der Film ist heute ein auch pädagogisch anerkanntes Medium, aber dennoch bleibt der Graben zwischen der medialen Alltagskultur von Kindern und Jugendlichen und einer pädagogischen Kultur, in die das Kino nicht hineingehört. Die Lernerfahrungen, die Kinder und Jugendliche dort machen, werden in der Pädagogik kaum reflektiert. Denn für Jugendliche bleibt das Kino ein doppelt positiv besetzter Ort, an dem nicht nur faszinierende Seh-Erlebnisse stattfinden, sondern ist auch ein jugendkultureller Ort der Geselligkeit mit Erlebnisqualitäten, die in der pädagogischen Welt nichts zu suchen haben. „Das Lernfeld Sehen und Emotionalität bleibt in seiner konzentriertesten Form dem Kino vorbehalten. »Vergnügen «, »Ablenkung«, »Faszination« oder »Spaß« sind keine Kategorien in pädagogischer Argumentation." (Baacke 1995, S. 83). 
Aus Untersuchungen zur Sozialgeschichte der Kindheit wissen wir, dass zur pädagogischen Domestizierung und Disziplinierung des Kindes auch gehört, dass das Auge des Kindes nur sehen darf, was der Pädagoge inm zu sehen erlaubt. Der Blick des Kindes wird gelenkt, denn jedes Sehen muss systematisch erfolgen und sich den Unterrichtsritualen unterwerfen. Aus der schwarzen Pädagogik (z.B. Rutschky 1977, S. 587 ff.) sind viele Beispiele bekannt, denen sich entnehmen lässt, wie das Sehen des pädagogischen Blicks zu sein hat. Baacke beschreibt das so:

Das Sehen des pädagogische Blick „ist gelenkt (durch den Lehrer/die Lehrerin); es ist imitativ (ahmt vorgemachtes Verhalten nach, verzichtet auf eigene Entdeckungen); kontrolliert (nur das darf ins Blickfeld geraten und gesehen werden, das für den Blick auch freigegeben ist); homogenisiert (alle sollen das Gleiche sehen, Abweichungen des Blicks sind nicht erlaubt). Dieser »pädagogische Blick « ist ein Blick, der den Adressaten verfehlt, auf den er sich richtet. So ist der pädagogische Blick aufs Kino und das in ihm Gebotene - im Gegensatz zum Vergnügen, dass das Kino Kindern und Jugendlichen bereitet - von Bedenklichkeiten getrübt und färbt den Ort grau in grau. Wir wissen, dass Unterschiede und Differenzierungen eines Gebiets oder Gegenstands von denen übersehen werden, die nicht kundig sind. Für Erwachsene, denen jugendkulturelle Erlebnismuster eher fremd sind und die innen entsprechend ablehnend gegenüberstehen, sind alle Jeans blau, alle Rock-Konzerte undifferenziert laut, alle Horrorfilme aggressiv, geschmacklos und unerträglich trivial. So verfehlt der pädagogische Blick viele Orte dieser Erde, an denen Jugendliche sich mit besonderer Vorliebe aufhalten, und er grenzt ihre kulturellen Interessen und Äußerungen entsprechend aus dem Feld aus, das er übersieht. In einer unbekannten oder geistig nicht bearbeiteten Situation drängen sich eben grobe Allgemeineindrücke derart vor, dass Unterschiedlichkeiten sich einebnen." (Baacke 1995, S. 84).

Daraus lässt sich folgern, dass Bildung und Film noch längst nicht versöhnt sind, ihr Verhältnis vielmehr pädagogisch erst entfaltet werden muss, so dass zum einen die emotionalisierenden Bilder auch Teil pädagogischer Lerngeschichte sind, der pädagogische 
Blick also Reichweite und Fokus verändern muss, um besser differenzieren und verstehen zu können, und andererseits das Sehen von Bildern auch als Teil des pädagogischen Auftrags verstanden wird. "Dies setzt voraus, dass Pädagogen Ahnung haben von optisch arrangierten Erlebnisformen und ihrer Bedeutung im Rahmen jugendlicher Sozialisation, und dass sie mit den Filmen etwas anfangen können, die Jugendliche sehen. Medienkundliches Wissen ist Voraussetzung für jede (medien-)pädagogische Einmischung, die sich nur dann legitimiert, wenn sie den Kommunikationskreis nicht zerstört, in den sie eindringt." (Baacke 1995, S. 85, Hervorhebung R.V.)

Eine Filmbildung ohne "pädagogischen Blick" beinhaltet im Übrigen keine Abkehr von Reflexion - wohl aber eine Rehabilitierung der sinnlichen Erfahrung, an die die Reflexion nun rückgebunden wird. Filmbildung in diesem Sinn ist Wahrnehmungsbildung wohlgemerkt Wahrnehmungsbildung und nicht die gelenkte Wahrnehmungserziehung des pädagogischen Blicks. Wer seine Wahrnehmung bildet, wird reich an Erfahrungsbildern, an Deutungsmöglichkeiten, die jeder für sich nach eigenen Zwecken, Zielen und Interessen ordnen, aber auch mit denen der anderen abstimmen muss.

Mit diesen Bemerkungen zu Baackes These vom pädagogischen Widerwillen gegen den Seh-Sinn will ich es erst einmal gut sein lassen, und wechsele die Perspektive auf Bildung. Ich hatte anfangs davon gesprochen, dass der Begriff Filmbildung offenlässt, wie diese beiden Aspekte - Film und Bildung - gewichtet werden. Dass dies ganz unterschiedlich ausfallen kann, zeigen die verschiedenen Konzepte von Filmbildung. Ich will hier mehrere Konzepte vorstellen.

\section{Der Filmkanon}

Dazu heißt es auf der Internet-Site der Bundeszentrale für politische Bildung: „Auf Einladung der Bundeszentrale für politische Bildung kamen im Juli 2003 Filmschaffende, Filmhistoriker, Filmkritiker und Filmpädagogen zusammen, um einen Filmkanon von 35 Filmen zu diskutieren und zu beschließen. Ziel war es, bedeutenden Werken der Filmgeschichte (Hervorhebung R. V.) auch im 
Schulunterricht mehr Aufmerksamkeit zu verschaffen und so der filmschulischen Bildung in Deutschland neuen Auftrieb zu geben. Der Kanon erhebt dabei nicht den Anspruch, einen vollständigen Überblick über das umfangreiche Schaffen der schon über 100jährigen Filmgeschichte zu geben. Vielmehr will er sensibilisieren für die Vielfältigkeit dieser Kunstform, für die Geschichte des bedeutendsten Mediums des 20. Jahrhunderts und für das Verstehen des Films der Gegenwart." (http://www.bpb.de/gesellschaft/kultur/filmbildung/filmkanon/)

Hinter dieser "filmschulischen Bildung" (auch eine interessante sprachliche Konstruktion) steht offensichtlich ein Bildungsbegriff, mit dem darauf abgezielt wird, die angehäuften Film-Schätze der letzten 100 Jahre an die nachfolgenden Generationen weiterzugeben. Dieses sind die "bedeutenden Werke der Filmgeschichte", die heutige Kinder und Jugendliche sich aneignen sollen. Das ist ein traditioneller Bildungsbegriff, der Hoch- und Trivial-Kultur säuberlich trennt - was in pluralistischen Gesellschaften als durchaus schwierig angesehen werden muss -, mit dem dann vermeintliche Bildungsgüter identifiziert und traditionalisiert werden, also an die nachfolgenden Generationen weitergegeben werden.

Das ist in hohem Maße Ideologie-anfällig und dient wohl auch der Elitenlegitimation. Denn Bildung in diesem Sinne ist zuvörderst ein Mechanismus der Distinktion, mit dem "der Gebildete", die "Hohe"-Kultur, die richtige Tradition und die "feinen Unterschiede" abgegrenzt werden können gegen all das, was die Definitionsmächtigen davon ausschließen. Da eine in sich homogene Bildungsschicht im Sinne dieses Bildungsbegriff aber nicht mehr existiert, ist ein solcher Kanon auch im Diskurs der "Gebildeten" äußerst umstritten - die damalige Debatte, die unter anderem in der "Zeit" viel Raum bekommen hat, spiegelte diese Unstimmigkeiten. Immerhin konnten viele Prominente in der Debatte ihre jeweiligen Befindlichkeiten äußern, auch wenn sie von Pädagogik nichts, aber auch gar nichts verstehen, wie vielen Leserkommentaren in der "Zeit" zu entnehmen war.

Allzu weit ist das Konzept nicht von den Vorstellungen Wilhelm von Humboldts entfernt, der vor 200 Jahren die Antike als Kanon empfahl: „Auch Griechisch gelernt zu haben, könnte auf diese 
Weise dem Tischler ebenso wenig unnütz seyn, als Tische zu machen dem Gelehrten." (von Humboldt 1809, S. 176). Das ist ja nicht falsch - ebenso wenig wie eine Auseinandersetzung mit der Geschichte des Films unnütz wäre -, aber vielleicht ist dies doch nicht die zentrale Aufgabe der heutigen Schule, und reicht sicherlich bei weitem nicht, den Herausforderungen durch neue mediale Bilderwelten, mit denen Kinder und Jugendliche heute konfrontiert sind, angemessen zu begegnen.

Der Kanon abstrahiert vom historisch-gesellschaftlichen Entstehungskontext der Filme und dem Rezeptionskontext der heutigen Kinder und Jugendlichen. Nicht aus filmgeschichtlicher, filmtheoretischer oder filmästhetischer, sondern aus pädagogischer Perspektive verfehlt er damit seinen Bildungsanspruch, und im schlechtesten Fall erwerben Kinder und Jugendliche damit nicht Bildung, sondern nur totes Konservenwissen. Bildung und demzufolge auch Filmbildung vollzieht sich immer in einer Gegenwart. Historische Bildungsstoffe müssen entsprechend anschlussfähig sein - das ist gerade für jüngere Zielgruppen aber nicht ohne Weiteres der Fall.

\section{Beispiel: „Emil und die Detektive" (1931)}

Das erste Drehbuch soll Erich Kästner sehr erbost haben: „Das Manuskript ist ekelhaft. Emil klaut in Neustadt einen Blumentopf für die Großmutter. In Berlin auf der Straßenbahn klaut er einem Herrn den Fahrschein aus dem Hut und lässt ihn für sich knipsen. Der Herr wird von der Bahn gewiesen. Ein Goldjunge, dieser Emil. Der »Stier von Alaska « wird er genannt und Pony die »Rose von Texas « [...]. Lauter Indianerspiele, wo heute doch keiner mehr Indianer spielt [...]. Die ganze Atmosphäre des Buchs ist zum Teufel. Ich werde Anfang der Woche saugrob werden." - so beschreibt es Uschi Reich ${ }^{1}$, die den Film auf der Internetsite der Bundeszentrale für politische Bildung zum Filmkanon vorstellt. Das Drehbuch wurde daraufhin massiv verändert. Das Ergebnis ist ein guter Film, gar keine Frage.

${ }^{1}$ Reich, Uschi 2010: Emil und die Detektive. URL: http://www.bpb.de/gesellschaft/kultur/filmbildung/filmkanon/43544/emil-und-die-detektive 
Dennoch scheint nach 80 Jahren die Kritik Kästners wieder treffend: "Lauter Indianerspiele, wo heute doch keiner mehr Indianer spielt." Die Darstellung einer Straßenkindheit im damaligen Berlin war treffend und sehr gelungen, aber für heutige Kinder ist das eine völlig fremde Welt, die sie nicht anspricht. Heute laufen Kinder mit dem Smartphone durch die Stadt und nicht mit der Hupe, und mittels Smartphone organisieren sie auch ihre Netzwerke und Freizeitaktivitäten. Wenn ein Zehnjähriger heute mit der Originalfassung von Emil und die Detektive aber wenig anfangen kann, weil seine eigenen lebensweltlichen Erfahrungen nicht anschlussfähig sind - wie soll der Film dann bilden? Allenfalls kann er historisches Filmwissen vermitteln, aber braucht es dazu eines Kanons, und ist das überhaupt das zentrale Problem schulischer Filmbildung? Ich meine nicht.

Kanonbildung impliziert auch Ontologisierung. Die Filme, die im Kanon sind, werden zum Maß aller Dinge erklärt, und umgekehrt andere Filme automatisch abgewertet, allein dadurch, dass sie nicht aufgenommen wurden. Das ist ein Problem, denn es begrenzt und fördert nicht die Lust auf eigene Erfahrungen. Und wer verantwortet überhaupt diesen Kanon? Die Bundeszentrale für politische Bildung als oberste Bildungsinstanz? Die 19-köpfige Expertenkommission, die den Kanon zusammengestellt hat, zu der laut mediaculture-online ${ }^{2}$ Regisseure, "weitere namhafte Filmwissenschaftler, Produzentinnen und Medienpädagogen", also offenbar weder Schulpädagogen noch Experten für Kinder und Jugendliche gehörten? Welchen Bezug haben diese Experten zu heutigen Kindern, ihren Lebenswelten, ihren Rezeptionsgewohnheiten und nicht zuletzt zur Schule, denn für diesen Kontext ist der Kanon ja gedacht?

Weiter heißt es auf www. mediaculture-online.de: „Natürlich löste der Filmkanon auch Kritik aus: Wurden zu viele gut »abgehangene« Klassiker gewählt? Warum ist keine einzige Regisseurin vertreten?" (ebd.). Letzteres Argument muss man sich auf der Zunge zergehen lassen. Als Auswahlkriterium für den Bildungswert eines Films wird hier ernsthaft das Geschlecht der Regisseurin bzw. Regisseurs (warum nicht auch Drehbuchautors, Autors

${ }^{2}$ http://www.mediaculture-online.de/Filmkanon.36.0.html 
der Buchvorlage oder Kamerafrau?) ins Spiel gebracht. Verstehen könnte ich, wenn danach gefragt wird, ob eine Geschichte aus männlicher oder weiblicher Sicht erzählt wird, oder ob ein Film eher Jungen oder Mädchen anspricht. In der Schulpraxis würde man allerdings wohl eher darauf achten müssen, dass er Jungen und Mädchen gleichermaßen anspricht - jedenfalls bei gemischtgeschlechtlichen Klassen.

Kaum berücksichtigt im Kanon sind Filme für Kinder unter zwölf Jahren, wie auch der Bundesverband Jugend und Film kritisierte. Der BJF hat daher mit der Fachzeitschrift "Kinder und Jugendfilm Korrespondenz" (KJK) auf Basis einer Umfrage unter 25 Kinderfilmexpertinnen und -experten einen ergänzenden Kinderfilmkanon mit Filmen für Sechs- bis Zwölfjährige erstellt ${ }^{3}$ - übrigens ebenfalls mit der Originalfassung von „Emil und die Detektive" von 1931.

In Sachsen beispielsweise werden Schülerinnen und Schüler flächendeckend durch "Schindlers Liste" geschleift. Wenn die pädagogische bzw. politische Absicht aber so plump daher kommt, versteht noch der letzte Schüler, dass und in welche Richtung seine Einstellungen verändert werden sollen. Diese „Bildungssituation" kann dann der beste Film nicht retten, und man muss sich nur einmal mit den Schülerinnen und Schülern unterhalten, um zu sehen, wie kontraproduktiv das in manchen (nicht allen) Fällen ist.

Festhalten lässt sich, dass das Kanon-Konzept Filmbildung ganz klar auf Film betont und auch den Entstehungskontext und die Entstehungszeit der ausgewählten Filme pädagogisch vernachlässigt, so dass die Filme oft nicht den Themen, Sehgewohnheiten und Lebenswelten heutiger Kinder entsprechen. Das liegt auch daran, dass mit der Idee eines Kanons eine Vorstellung von Bildung verbunden ist, die Bildung nicht als Prozess, sondern als Substanz versteht. In der Pädagogik ist heute dagegen ein prozessualer Bildungsbegriff vorherrschend. Dabei geht es um die Prozesse, in denen Subjekte sich bilden - Bildung als Selbstkonstruktion des Subjekts - und wie sich solche Prozesse pädagogisch befördern lassen - beispielsweise durch Schaffung von Bildungsgelegenhei-

${ }^{3}$ KJK 2004: Filmkanon für Kinder (Sonderheft), 2. Auflage 2007 
ten. Eine solche Bildungsgelegenheit kann die Präsentation, Diskussion und Auseinandersetzung mit Filmen immer dann sein, wenn sie anschlussfähig ist an Themen, Entwicklungsaufgaben, Wissen, Erfahrungen, Interessen und Bedürfnisse der Subjekte.

So löblich die Absicht ist, Filmbildung in der Schule stärker zu verankern, so problematisch ist ein Kanon, zumal in so stark filmgeschichtlicher Ausrichtung. Gäbe es eines Tages eine Computerspiel-Bildung, und einen Computerspiel-Kanon, müssten die Kinder sich dann vermutlich mit Super Mario in Arcade-Spiel-Ästhetik als Highlight der achtziger Jahre auseinandersetzen.

Einen weniger traditionellen, allerdings unkonkreten Bildungsbegriff hat beispielsweise von Daniel Goeudevert (1981) formuliert, der Bildung als einen "aktiven, komplexen und nie abgeschlossenen Prozess (beschreibt/R. V.), in dessen glücklichem Verlauf eine selbstständige und selbsttätige, problemlösungsfähige und lebenstüchtige Persönlichkeit entstehen kann". Ziel von Bildungsprozessen ist dann nicht "Wissen" - dieses ist vielmehr selbst nur Hilfsmittel für Bildung, die auch Reflexion und kritische Distanz, mithin Urteilsvermögen voraussetzt. Angesichts pluraler Normen und Werte geht es in heutigen Bildungswelten nicht um die inhaltliche Weitergabe von Wissen, sondern um Begriffspaare wie "Kompetenz und Teilhabe", "Distanz und Kritik", "Kreativität und Innovation" oder "Universalität und Eigensinn" (vgl. Tenorth: Bildung im Wandel - Vortrag, Uni Marburg, 18.4.2012). Eine aktuelle Definition von Klieme u. a. von 2003 geht vor dem Hintergrund der Kompetenzdebatte noch weiter. In den sogenannten "nationalen Bildungsbildungsstandards" heißt es „»Bildung « ... bezeichnet ... die Fähigkeit von Subjekten, unter den Bedingungen der Unentscheidbarkeit und Unbestimmtheit, Offenheit und Pluralität handlungsfähig zu sein (und ) ... mündig zu werden, ... so dass "Gleichheit und Individualisierung ... zugleich" eröffnet werden." (Klieme et al. 2003, S. 65).

Wir sehen, die Bildung-Vorstellungen sind sehr verschieden: von Substanz über Prozess zur Fähigkeit. Das kann ich hier leider nicht weiter vertiefen. Stattdessen möchte ich fünf weitere Konzepte von Filmbildung beispielhaft anführen, die an der Fakultät Erziehungswissenschaften der Technischen Universität Dresden tatsächlich alle vorzufinden sind. 


\section{Cineastisches Konzept}

Cinéaste bezeichnet im Französischen noch heute einen Filmregisseur, während der Begriff im Deutschen vor allem auf die Differenz zwischen Kommerz-Kino und einem Kino mit Kunstanspruch verweist. Ein Cineast ist ein Mensch, der als Filmliebhaber beschrieben werden kann, als ausgewiesener Kenner von Filmkunst und Filmgeschichte, der auch bestens vertraut ist mit der Formen- und Motivgeschichte des Films und zudem Interesse am Filmdiskurs hat.

Filmseminare für Cineasten vermitteln Expertenwissen. Sie haben beispielsweise Titel wie "Der russische Avantgarde-Film von 19081934". Auch ein cineastisches Konzept ist elitär. Während beim Filmkanon aber die alten Bildungseliten ihre Filmbildungsideale auf heutige Kinder und Jugendliche insgesamt projizieren, bleiben $\mathrm{Ci}-$ neasten weit gehend unter sich. Cineastisches Filmbildung ist Filmbildung für Cineasten. Sie verfehlt daher keineswegs ihre Zielgruppe, sondern bietet ihr außergewöhnliche Bildungsgelegenheiten und fördert insgesamt den Diskurs über Filme. Insofern ist sie auch unverzichtbar. Sie ist nur sehr speziell und daher für die Schule wenig geeignet.

\section{Studentisches Kino}

An der TU Dresden gibt es das „Kino im Kasten", das seit 20 Jahren von Studierenden betrieben wird. Hier geht es um Bildungserfahrungen ganz anderer Art. Studierende, die selber ein Kino betreiben, müssen ihre Filmerfahrungen reflektieren und transzendieren, denn die gezeigten Filme sollen ja ein Publikum ansprechen und nicht nur sie selbst. Die studentischen Kinobetreiber haben selbst oft hohe Ansprüche, sind meist Cineasten, bekommen aber zu spüren, dass ein zu elitäres Programm nicht massenkompatibel ist. So kann man beobachten, dass in einer Saison (gerade wenn wieder eine neue Gruppe Verantwortung übernommen hat) ein sehr anspruchsvolles Programm gezeigt wird, dem dann im nächsten Semester - weil offenbar zu wenig Zuschauer kamen - ein 
vom Anspruch her anders austariertes Programm folgt. Die Studierenden lernen, dass man sich ein Publikum heranbilden muss und das ist selbstverständlich auch wieder ein Bildungsprozess.

\section{Lehrfilme}

Ein weiterer Ansatz, der bei uns an der TU Dresden in der Bildungstechnologie verankert ist, ist die Produktion kurzer Lehrfilme durch Studierende. Dabei geht es darum, dass Studierende in kleinen Gruppen das Handwerkszeug erwerben, einen kleinen eigenen Lehrfilm zu produzieren. Hier liegt der Schwerpunkt eindeutig auf der Didaktik, also bei der Frage, wie man einen bestimmten, konkreten Inhalt in einem Film didaktisch so aufbereiten kann, dass Zuschauer einen möglichst hohen Lernerfolg haben. Auch hier lernen Studierende nebenbei eine Menge über das Medium Film, über Gestaltungsmittel, über Schnitt, über Kameraführung etc. Die Perspektive ist jedoch eine ganz andere als bei der Auseinandersetzung mit Spiel- oder Dokumentarfilmen.

\section{Handlungsorientierte Medienpädagogik}

In Seminaren zur handlungsorientierten Medienpädagogik können Studierende lernen, eine Internet-Site zu programmieren, oder ein Hörspiel oder einen Film zu produzieren. In kleinen Gruppen müssen Sie ein Thema finden, ein Drehbuch schreiben und den Film dann auch drehen. Zuvor erhalten Sie eine ausführliche Einführung, die theoretische Elemente ebenso umfasst wie ganz praktische Hinweise. Die Gruppen werden durchgängig von Tutoren betreut. Zum Schluss werden die Filme gezeigt und besprochen, Probleme diskutiert und auch Verbesserungsvorschläge gemacht. In die Leistungsbewertung fließt dann auch eine schriftliche Reflexion der Seminarteilnehmer ein.

Ebenso wie bei den Lehrfilmen geht es offensichtlich nicht um rezeptive, sondern um aktive Medienarbeit. Statt Filmanalyse und Filmkritik geht es hier um eigene Gestaltung, ästhetische Entscheidungen, die Frage, wie ich bestimmte Wirkungen erzielen kann, indem ich bestimmte filmische Gestaltungsmittel einsetze usw. Damit werden andere Dimensionen von Medienkompetenz 
angesprochen als bei der rezeptiven Medienarbeit. Auch hier ist eine theoretische Auseinandersetzung mit dem Medium und seinen Möglichkeiten notwendig, aber auch eigene Kreativität gefragt. Nicht zuletzt ist auch die Erfahrung des eigenen Tuns wesentlich - gerade in einem Bildungssystems, das ansonsten stark auf die Aufnahme und Verarbeitung von Wissen ausgerichtet ist.

\section{Der Spielfilm als Lernfeld narrativer Pädagogik}

In meinen Filmseminaren, die ich die lange Zeit zusammen mit Horst Schäfer, dem langjährigen Leiter des Kinder- und Jugendfilmzentrum der Bundesrepublik (KJF) durchgeführt habe, erlernen Studierende zunächst Methoden der Filmanalyse und erwerben Grundkenntnisse über Gestaltungselemente des Films und deren Wirkungen auf Zuschauer, insbesondere auf Kinder und Jugendliche (s. den Artikel "Filmsprache und Filmanalyse" von Horst Schäfer). In einem drei- bis viertägigen Block stellen die Studenten dann ihre medienpädagogischen Analysen von Filmen vor, die jeweils unter einem spezifischen Seminarthema stehen. Dabei wird zunächst der Film gezeigt, und im Anschluss an das Referat (oft mit Ko-Referaten zu spezifischen Aspekten oder beispielsweise einem Vergleich des Film mit einer Literaturvorlage) findet eine meist sehr lebhafte Diskussion dazu statt.

Die Intention dieser Seminare, die auf eine lange Tradition zurückgehen und schon von Dieter Baacke und Horst Schäfer konzipiert, freilich auch immer weiter entwickelt wurden, ist jedoch grundsätzlicher und hochschuldidaktischer Natur. Die Frage ist doch, wie wir als Lehrende damit umgehen können, dass Studierenden pädagogisches Theoriewissen oft sehr praxisfern erscheint, und wie man ihnen dieses anders nahe bringen kann. Eine Antwort darauf gibt das von Dieter Baacke und Theodor Schulze im Rahmen der pädagogischen Biographieforschung entwickelte Konzept "Aus Geschichten lernen" (Baacke/Schulze 1979). Im Kontext dieser Überlegungen ist auch Dieter Baackes Seminarkonzept zum "Spielfilm als Gegenstand pädagogischer Analyse" (s. den Artikel von Baacke in dieser Ausgabe) zu sehen. Spielfilme enthalten sehr viele pädagogisch relevante Anknüpfungspunkte wie beispiels- 
weise Entwicklungsthemen, Beziehungen, Konflikte und Konfliktlösungen etc. - aber eben nicht wie in einem theoretischen Lehrbuch fein säuberlich analytisch aufbereitet und dementsprechend abstrakt und von lebensweltlichen Bezügen entkernt, sondern in der narrativen Eingebundenheit der handelnden Personen. Entscheidend ist gerade diese Narration, da sie lebensweltliche Nähe herstellt, Sinnlichkeit zulässt, Zusammenhänge erkennbar werden lässt, das Handeln von Personen in soziale und biographische Kontexte stellt, aber auch widersprüchlich und widerständig sein kann und womöglich gerade darin authentisch.

In der medienpädagogischen Filmanalyse geht es nun darum, die relevanten pädagogischen Aspekte analytisch herauszuarbeiten und unter Hinzuziehung entsprechender wissenschaftlicher Literatur einzuordnen und zu deuten (siehe dazu meine Beispielinterpretation über Typen der Zeiterfahrung von Jugendlichen im Film "Das Ende des Regenbogens" in dieser Ausgabe). Über Spielfilme gelingt ein ganz anderer Zugang zu pädagogischen Thematiken, als Studierende inn sonst gewohnt sind. Es fällt innen in der Regel auch leichter, Theorie und Praxis zusammen zu denken, und das didaktische Problem, dass Wissen quasi auf Vorrat gelernt wird, bis es in einer Klausur oder Hausarbeit abgefragt wird, ist bei dieser Seminarform deutlich geringer. Studierende liefern in diesen Seminaren Arbeiten in hoher Qualität ab (s.a. die Magisterarbeit von Uta Quietzsch über "Sterben und Tod in Kurzfilmen für Kinder" in dieser Ausgabe), und ihre Seminarrückmeldungen sind manchmal überwältigend - positiv. Für die Studierenden steht die Auseinandersetzung mit dem Film übrigens oft derart im Vordergrund, dass sie die pädagogische Absicht der Vermittlung pädagogischen Wissens gar nicht bemerken, obwohl sie für ihr Referat auch wissenschaftliche Literatur hinzuziehen.

Viele der Schwierigkeiten solcher Filmseminare, die Dieter Baacke bereits in seinem Aufsatz "Der Spielfilm als Gegenstand pädagogischer Analyse" von 1979 genannt hat, sind heute unverändert. Zwar ist die Filmbeschaffung dank DVD leichter geworden, aber technische Probleme gehören auch heute noch zum Alltag. Auch sind die Universitäten noch immer weder räumlich noch zeitlich auf solche Lehrformen eingerichtet. An der TU Dresden beispiels- 
weise können solche Blockveranstaltungen (die wegen der Filmlänge als Block alternativlos sind) aufgrund dafür fehlender Räume nur in der vorlesungsfreien Zeit durchgeführt werden, da im „Zeitregiment" ansonsten kein Platz ist. Im Anschluss an die Vorlesungszeit sind geeignete Räume - Vorlesungsräume mit Verdunkelungs- und Projektionstechnik - aber dank BA/MA-System flächendeckend für Klausuren reserviert, sodass man in die bei Studenten eher unpopulären Ferienzeiten (die für sie oft auch Arbeitszeiten sind) ausweichen muss. In manchen Semestern ist es mir glücklicherweise (und mit sehr viel Aufwand) gelungen, Kooperationspartner in der Stadt zu finden, die uns ihre Räume für ein Filmseminar zur Verfügung gestellt haben. Dafür möchte ich mich an dieser Stelle bedanken bei den Verantwortlichen vom Medienkulturzentrum e.V., dem Deutschen Hygiene-Museum, den Technischen Sammlungen Dresden, dem kid - Kino im Dach, dem Kino im Kasten und dem Thalia-Filmtheater.

Eine inhaltliche Herausforderung besteht heute vor allem bei den Studierenden der Lehramtsstudiengänge, die oft eine verkürzte Perspektive auf die Einsatzmöglichkeit der gezeigten Filme für ihren eigenen Unterricht haben (und dann zu dem Schluss kommen, dass der Film zwar ganz gut, aber zu lang ist oder dass das Thema für ihre Klassenstufe nicht passt). Gegen solche Funktionalisierungen ist - neben dem Aspekt des exemplarischen Lernens - auf der Sinnlichkeit des Films zu bestehen, der in erster Linie ein ästhetisches Erlebnis ist. Dies ist geradezu ein Argument für den Einsatz von Filmen in der Schule, aber Lehrerinnen und Lehrer müssen auch mit dieser Sinnlichkeit des Films umgehen können, statt sie zugunsten ihres Unterrichtsthemas wegzublenden.

Während das Kanon-Konzept ganz vom Film her konzipiert ist, ist das Konzept des Spielfilms als Lernfeld narrativer Pädagogik von der Pädagogik her gedacht. Dennoch geht es auch hier um „Filmbildung", nicht nur weil - gewissermaßen als Nebenintention - Filmanalyse und Filmkritik gelernt werden und viel filmspezifisches Wissen erworben wird, sondern weil auch die Filmreihe eines Seminars unter bestimmten filmischen Auswahlaspekten zusammengestellt wird, die sich nicht unmittelbar aus der pädagogischen Thematik ergibt. So wird beispielsweise auch ein Genrewissen vermittelt - etwa zum Horrorfilm, zum Fantasy-Film oder zum 
japanischen Animationsfilm. Eine kleine Übersicht der Seminartitel der letzten Jahre (in kalendarischer Reihenfolge) verdeutlicht solche inhaltlichen Klammerungen:

- Junges europäisches Kino (z.m. ARTE)

- "Top-Videos" für Kinder (Filmempfehlungen des KJF)

- Einführung in die Filmanalyse

- Jugendkulturen und Film

- Vorurteile, Aggressionen, Gewalt

- Horror! Klassik, Kult und Trash

- Animationsfilm

- Fantasy für Kinder

- Cyborgs - Menschmaschinen - Maschinenmenschen

- Nord-Licht-Bilder (Highlights des skaninavischen Kinder- und Jugendfilms)

- Kinder, Krieg und Kino

- Jugend in Deutschland

- Mythos Großstadt

- Das Kino des Phantastischen \#1 Schlaf und Träume

- Das Kino des Phantastischen \#2 Alpträume

- Dialog der Kulturen

- Helden - von Achill bis Zidane

- Kinderfilme mit Qualitätsansprüchen

- Mobile Movies (aktive Filmarbeit mit Handys)

- Jugendfilme mit Qualitätsanspruch

- Schule im Film

- Pädagogik im Film

- Comic - Kult - Filme

- Jugend unter dem Hakenkreuz

- Identität, Respekt und Anerkennung

- Filmanalyse und Interpretation von Literaturverfilmungen: Science-Fiction und Fantasy

- Die Welt aus den Fugen: Kritische Lebensereignisse im Kindes- und Jugendalter

- Blutsauger und Herzensbrecher: Vampirfilme im Wandel der Zeiten

- Der Dschungel ruft

\section{Filmbildung und Filmverstehen}

Das war ein kurzer Durchgang durch sechs unterschiedliche Konzepte von Filmbildung und sicherlich sind weitere Formen denkbar. 
Dazu gehören heute auch Internetportale wie die Kinderfilmwelt (www.kinderfilmwelt.de) und insbesondere ist auch an Filmfestivals zu denken wie den "Goldener Spatz" (Gera) der Kindermedienstiftung oder das vom Kinder- und Jugendfilmzentrum (KJF) veranstaltete Bundesfestival "Deutscher Jugendvideopreis" und "Video der Generationen", an denen Kinder und Jugendliche als Filmemacher oder auch in einer Kinderjury teilnehmen, ihre Arbeiten präsentieren und auch von Gleichaltrigen - ebenso wie im Austausch der Generationen - lernen können.

Der Film kann einerseits als Unterrichtsstoff, als Lerngegenstand betrachtet werden. Dabei wird dann mit pädagogischem Blick geschaut, und gesichertes und eindeutiges Wissen über den Film, seine Geschichte, seine Sprache und seine Wirkung angeeignet. Auf den Nachteil solcher Filmbildung hat auch Walberg hingewiesen: „Der »Dialog « mit dem Film und das Filmerleben treten dabei in den Hintergrund und werden im ungünstigen Fall sogar verhindert." (Walberg 2007, S. 32).

Aus theoretischer Sicht zu hinterfragen ist auch, dass viele Ansätze der Filmbildung auf einem zeichentheoretischen oder semiotischen Filmverständnis basieren, demzufolge Bilder bekanntlich Zeichen sind, die auf etwas anderes verweisen. „Film verstehen" setzt dann voraus, die filmischen Codes zu kennen, mittels derer Bedeutung erzeugt wird. Kinder und Jugendliche sollen diese Regeln lernen - vorzugsweise durch Filmanalyse und Filmkritik - und in Kenntnis dieser Regeln dann auch weniger anfällig für Manipulationen sein. Auf dieser Denkfigur basiert meist auch die Forderung nach Filmbildung in den Schulen. Aus filmtheoretischer Sicht ließe sich dagegen kritisch einwenden, dass ein solches Filmverständnis filmische Innovationen nicht angemessen erfassen kann, da "die Analysierbarkeit von Botschaften gerade auf der Konventionalität der verwendeten Codes beruht" (Walberg 2007, S. 34). Aus pädagogischer Sicht ließe sich kritisch einwenden, dass die Forderung, Filme kritisch und distanziert zu analysieren, diese zu einem bloßen Werkzeug macht, um die Fähigkeit zum Filmverstehen zu vermitteln.

„Diese Konzentration auf Analyse und Verstehen kann Bildungsprozesse verhindern. Denn bei der Vermittlung eines analytischen Handwerkszeugs geht es ja um die Fähigkeit, 
den Film »in den Griff zu bekommen «, inn zu verstehen, zu entschlüsseln, ihn so zu zerlegen, dass er sich in vorhandene Kategorien des Selbst- und Weltverhältnisses einordnen lässt. Vorhandene Selbst- und Weltverhältnisse werden durch diesen Zugriff eher stabilisiert, als zur Disposition gestellt." (Walberg 2007, S. 34).

Freilich bleiben Filmanalyse und Filmkritik für die Filmbildung unverzichtbar, denn erst die Fähigkeit zu Analyse und Kritik auf der Basis grundlegenden Filmwissens, ermöglicht es, Filme auch kreativ und unkonventionell zu interpretieren. Statt Festlegungen im pädagogischen Blick braucht Filmbildung jedoch die Bereitschaft, solche Deutungsspielräume offen zu halten, die Filme mehr noch mehr als Texte bieten.

Ziel einer Filminterpretation sollte daher nicht sein, dass hinterher alle einer Meinung über den Film sind, sondern dass die vielfältigen Möglichkeiten aufgezeigt werden, wie der Film oder bestimmte Aspekte des Films erlebt und konsistent interpretiert werden können. Es ginge also darum, über die Weltwahrnehmung im Film zur Differenz zu gelangen statt zur übergreifenden Parole. Dann muss eine Filminterpretation auch nicht das Filmerleben zerstören - mit dieser Faszination, die viele Filme ausstrahlen, mit ihren Entrückungen in Traumwelten, den Spiegelungen zwischen Identifikationen und Projektionen, die unseren Alltag transzendieren.

An den Abschluss meiner Überlegungen möchte ich ein Zitat von Dieter Baacke stellen, für den Faszination der Schlüsselbegriff von Filmbildung ist:

"Nicht der Film in seiner fragwürdigen oder avantgardistischen Selbstreferenz, sondern seine Wirkung auf den zuschauenden, affizierten Betrachter steht im Mittelpunkt. Erlauben wir Faszination, kann ästhetische Erfahrung nicht mehr als Banausentum verpönt werden, und nicht notwendig muss sie sich aus der Sphäre des Genusses zu der ästhetischen Augenschein analysierenden Reflexion erheben auf dem Grunde pädagogischer Anleitung. Der Rezeptionsvorgang selbst wird dem Betrachtenden frei verfügbar gemacht und damit seinem je spe- 
zifischen kulturellen Habitus. Mit »Faszination « wird als möglich gedacht, dass das kindliche oder jugendliche Subjekt auch außerhalb gelenkter pädagogischer Verarbeitungshilfen seine ästhetischen Erfahrungen machen kann, deren Ausmünzung auf die Lebensrealität außerhalb des Films nicht pädagogisch eingeholt werden kann, sondern dem Subjekt selbst zur Disposition steht. Was ist das anderes als Bildung?" (Baacke 1995, 89). 


\section{Literatur}

Baacke, Dieter 1979: Der Spielfilm als Gegenstand pädagogischer Analyse. In: FEOLL - Schriftenreihe ,Universitäre Medieninformation', H. 7, S. 11-48, Paderborn.

Baacke, Dieter 1995: Zum pädagogischen Widerwillen gegen den Seh-Sinn. In: GMK-Rundbrief, Nr. 37/38, S. 80-94.

Baacke, Dieter/Schulze, Theodor (Hg.) 1979: Aus Geschichten lernen. Zur Einübung pädagogischen Verstehens. München.

Goeudevert, Daniel 1981: Der Horizont hat Flügel. Die Zukunft der Bildung. München.

Humboldt, Wilhelm von 1809: Königsberger Schulplan, Werke Bd. IV, S. 176.

Klieme, Eckhard et al. 2003: Zur Entwicklung nationaler Bildungsstandards. Eine Expertise. Bundesministerium für Bildung und Forschung, Juni 2003.

Reich, Uschi 2010: Emil und die Detektive. URL: http://www.bpb.de/gesellschaft/kultur/filmbildung/filmkanon/ 43544/emil-und-die-detektive).

Rutschky, Katharina (Hg.) 1977: Schwarze Pädagogik. Quellen zur Naturgeschichte der bürgerlichen Erziehung. Frankfurt.

Schultze, Ernst 1911: Der Kinematograph als Bildungsmittel. Halle.

Walberg, Hanne 2007: Film-Bildung an den Grenzen des Verstehens. Bildungstheoretische Überlegungen am Beispiel Jugend im Film. In: Decke-Cornill, Helene/Luca, Renate (Hg.): Jugendliche im Film - Filme für Jugendliche. Medienpädagogische, bildungstheoretische und didaktische Perspektiven. München, S. 31-43. 